\title{
Wireless Sensor Networks-A Review
}

\author{
Vahid Houshyarifar \\ Electrical and Electronics Engineering Department, \\ University Pardis of Urmia University, Urmia, Iran
}

\author{
Mehdi Chehel Amirani \\ Electrical and Electronics Engineering Department \\ Urmia University, Urmia, Iran
}

\begin{abstract}
This paper introduces the wireless sensor networks (WSNs) as an ad-hoc network and their structure in general and provides a small survey on sensors (nodes) as an embedded mechanism. The survey mainly focuses on the main challenges of these networks, such as the network layers of WSNs, data transfer over WSNs, the approaches for routing and packet management methods and localization of nodes.
\end{abstract}

\section{General Terms}

Wireless sensor networks, ad-hoc networks

\section{Keywords}

Routing, Mac, Localization.

\section{INTRODUCTION}

Recent advances in sensor and wireless communication technologies in conjunction with developments in microelectronics have made available a new type of communication network made of battery-powered integrated wireless sensor devices. Wireless Sensor Networks (WSNs), as they are named, are self-configured and infrastructure less wireless networks made of small devices equipped with specialized sensors and wireless transceivers [1]. The main goal of a WSN is to collect data from the environment and send it to a reporting site where the data can be observed and analyzed. Wireless sensor devices also respond to queries sent from a "control site" to perform specific instructions or provide sensing samples. Wireless sensor devices can be equipped with actuators to "act" upon certain conditions. These networks are sometimes more specifically referred as Wireless Sensor and Actuator Networks. At present time, due to economic and technological reasons, most available wireless sensor devices are very constrained in terms of computational, memory, power, and communication capabilities (Figure 1). This is the main reason why most of the research on WSNs has concentrated on the design of energy and computationallyefficient algorithms and protocols, and the application domain has been restricted to simple data-oriented monitoring and reporting applications. However, all this is changing very rapidly $[2,3,4]$, as WSNs capable of performing more advanced functions and handling multimedia data are being introduced. New network architectures with heterogeneous devices and expected advances in technology are eliminating current limitations and expanding the spectrum of possible applications for WSNs considerably.

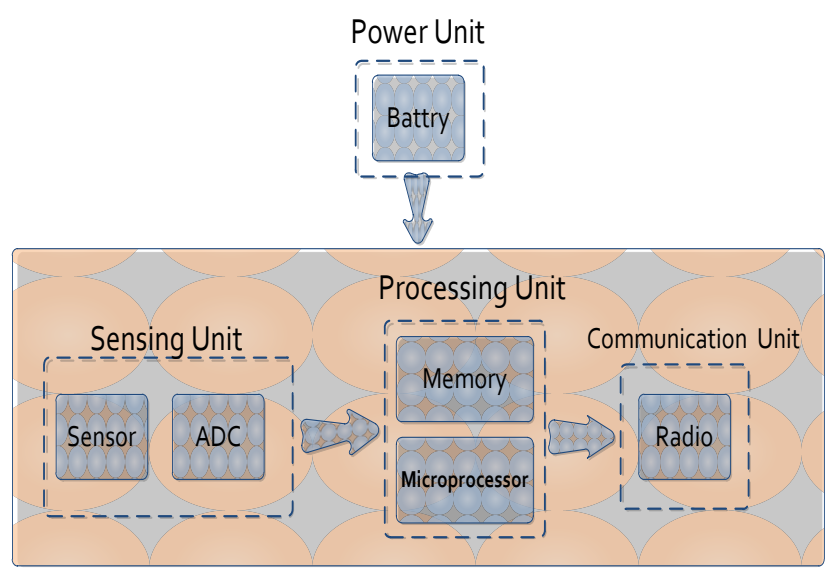

Fig 1: A typical sensor node hardware architecture

\section{RESEARCH CHALLENGES}

The main factors that complicate the protocol design for WSNs can be summarized in:

Fault tolerance: To sustain a network of WSN's after an error or fault causes in one or some sensors.

Power management: New methods in battery design and software challenges to manage this energy and how to administrate in a network. Hardware and software constraints originate a lot of design issues that must be addressed to achieve an effective and efficient operation of WSNs. Besides, new application scenarios lead to new challenges [5].

Location discovery: some applications to discover a sensor location with routing algorithms.

Cost: Wireless systems designers are being asked to provide low-cost, high-performance, easily deployed wireless sensors networks that change the way business processes are managed and monitored.

Some issues in WSN, can be broadly classified into three groups [6], namely, node system, middleware services, and communication protocols, as shown in Figure 2. In what follows, we simply outline some key research issues. 


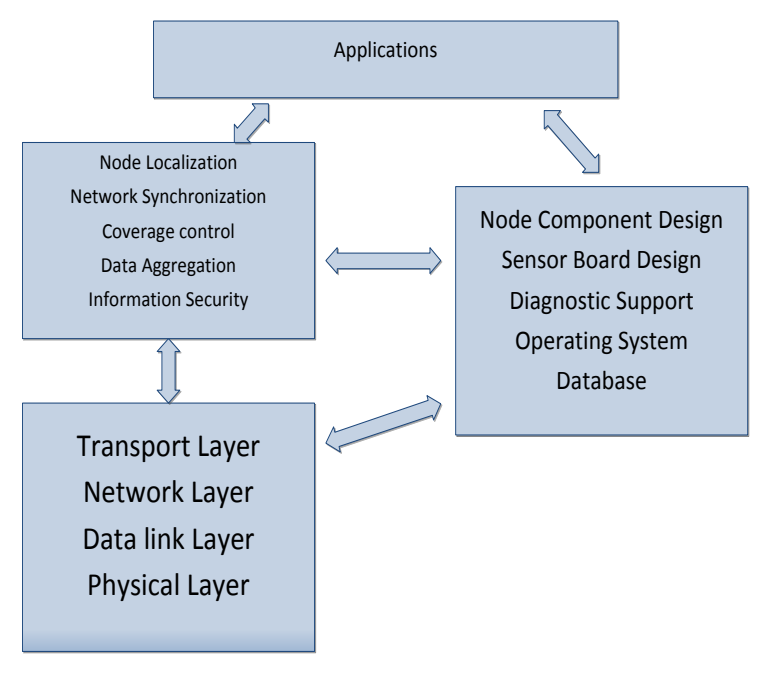

Fig 2: A categorization of key research issues in sensor networks

\begin{abstract}
Node System: Sensors measure physical data of the parameter to be monitored. The continual analogue signal produced by the sensors is digitized by an analogue-to-digital converter and sent to controllers for further processing. A sensor node should be small in size, consume extremely low energy, operate in high volumetric densities, be autonomous and operate unattended, and be adaptive to the environment.
\end{abstract}

Communication Protocols: The lower four layers of the communication protocol stacks are the physical layer, data link layer, network layer, and transport layer[7,8,13].

\section{MEDIUM ACCESS CONTROL}

MAC Protocols [9]: Medium access control has been extensively studied for traditional wireless networks. A variety of MAC protocols have been proposed to address different network scenarios. From different perspectives, MAC protocols can be classified into different categories, for example, centralized and distributed, single-channel based and multiple-channel based, contention based and contention free, and so on. Time division multiple access (TDMA), frequency division multiple access (FDMA), code division multiple access (CDMA), and carrier sense multiple access (CSMA) are typical MAC protocols that have been widely used in traditional wireless networks. However, these protocols do not take into account the unique characteristics of sensor networks, for example, denser levels of node deployment, higher unreliability of sensor nodes, and severe power, computation, and memory constraints. For this reason, traditional MAC protocols cannot be applied directly to sensor networks without modification. To design an efficient MAC protocol for sensor networks, the unique characteristics of sensor networks, in particular, energy efficiency and network scalability must be taken into account. Moreover, delivery latency, network throughput, bandwidth utilization, and fairness, which are the primary concerns in traditional wireless networks, should also be considered, but are of secondary importance in sensor networks . A MAC protocol must also take into account many factors in its design in order to improve network performance and provide good network services for different applications. Some of MAC protocols described below:

Sensor MAC (S-MAC) [11]: is an energy efficient MAC protocol specifically designed for WSNs. The primary goal of the S-MAC design is to improve energy efficiency while maintaining good scalability and collision avoidance. To achieve this goal, S-MAC tries to reduce energy consumption from all the major sources that cause inefficient use of energy. In exchange, it allows some performance degradation in both per-hop fairness and latency. This is implemented by integrating several effective control mechanisms into a contention - based MAC protocol built on top of the IEEE 802.11 standard. These mechanisms include periodic listen and sleep, collision avoidance, coordinated synchronization, and message passing. To reduce idle listening, S-MAC introduces a periodic listen and sleep mechanism to establish a low-dutycycle operation on each node. With this mechanism, each node is periodically put into a sleep state for some time, and then wakes up and listens to see if it needs to communicate with any other node.

Timeout MAC (T-MAC) [12]: In T-MAC, each node periodically wakes up to communicate with its neighbors and then go to sleep until the next frame. The nodes communicate with each other following a RTS-CTS-Data-ACK sequence, which provides both collision avoidance and reliable transmission. A node keeps listening and potentially transmitting as long as it is in an active period. If no activation event occurs for a threshold time $T_{h}$, an active period will end and the node will go to sleep. An activation event can be (1) the timing out of a periodic frame timer; (2) the reception of a data packet on the radio; (3) the sensing of communication on the radio; (4) the end of transmission of a node's own data packet or acknowledgment; or (5) the end of transmission of a neighbor's data packet. Obviously, $T_{h}$ determines the minimum amount of idling listening per frame. As a result, all nodes transmit at the beginning of each active period. Since data packets between active periods need to be buffered, the buffer capacity deter-mines an upper bound on the maximum frame time. The simulation results show that $\mathrm{T}$ MAC and S-MAC achieve similar energy consumption reductions (up to 98\%) compared to CSMA. However, TMAC outperforms S-MAC by a factor of 5 in a sample scenario with variable traffic load.

\section{ROUTING}

In small sensor networks where sensor nodes and a gateway are in close proximity, direct (single-hop) communication between all sensor nodes and the gateway may be feasible [14,15]. However, most WSN applications require large numbers of sensor nodes that cover large areas, necessitating an indirect (multi-hop) communication approach. The key responsibility of the network layer is to find paths from data sources to sink devices (e.g., gateways). In the single-hop routing model , all sensor nodes are able to communicate directly with the sink device. This direct communication model is the simplest approach, where all data travels a single hop to reach the destination. However, in practical settings, this single-hop approach is unrealistic and a multi-hop communication model must be used. In this case, the critical task of the network layer of all sensor nodes is to identify a path from the sensor to the sink across multiple other sensor nodes acting as relays.

\section{NODE LOCALIZATION}

Node localization is an enabling technology for WSNs because sensor nodes deployed in an area of interest usually need position information for routing and application-specific tasks, for example, temperature and pressure monitoring [16]. In many applications, a WSN is deployed to help improve localization accuracy in environments where the channel condition poses a challenge on range estimation [17]. In such environments, cooperative localization provides a potential for 
many applications in the commercial, public safety, and military sectors $[17,18]$. In commercial applications, there is a need for localizing and tracking inventory items in warehouses, materials and equipment in manufacturing floors, elderly in nursing homes, medical equipment in hospitals, and objects in residential homes. In public safety and military applications, however, indoor localization systems are needed to track inmates in prisons and navigate policemen, fire fighters, and soldiers to complete their missions inside buildings [18]. Node localization plays an important role in all these WSN applications. In general, a localization system incorporates range measurements to determine the location estimate. Figure 3 illustrates a block diagram of the main components in a traditional localization system. Essentially, the process for obtaining a location estimate involves different levels of complexities [19]. At the physical layer, the mobile terminal (MT) or the sensor node transmits and receives a waveform. From this radio frequency (RF) waveform, it is possible to extract the relevant range measurements. In RSS systems, for example, the total signal energy that a node/MT receives from an anchor/reference point (RP) can be used to estimate the distance. For a given received power, it is possible to estimate the corresponding distance with some certainty. The RSS technique is usually simple to implement, but suffers from inaccuracies, especially in multipath-rich environments. On the other hand, for TOA based systems, the distance is estimated by sending an RF signal and recording the time it takes to receive it. This approach is more accurate because the arrival time corresponds to the direct path distance. Once 3(4) range measurements are obtained from different anchors/RPs, the node/MT passes this information to a positioning algorithm, where the two - or three - dimensional (2D or 3D) position is then estimated. The range measurements essentially constrain the possible location of the MT. The area of uncertainty of a location estimate decreases as the accuracy of range measurements improves. Figure 4 [19] shows an example of 2D localization, where a node/MT has three range measurements to different anchors/RPs. The positioning error is affected by the accuracy of the range measurements, the number of anchors/RPs, and their relative geometry to the sensor node/MT. Finally, the estimate of the location is displayed to the user with information regarding its quality or accuracy.

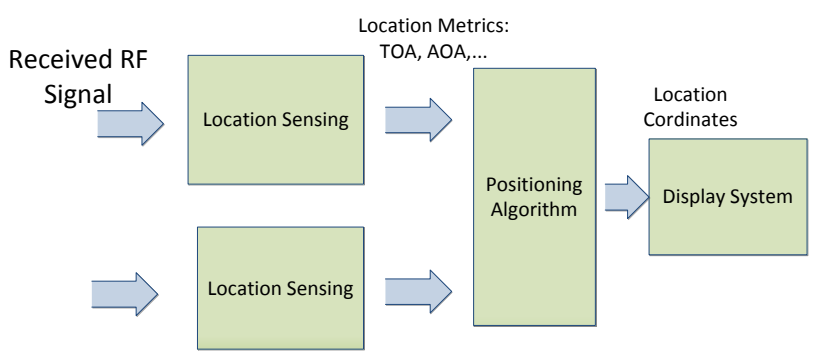

Fig 3: Localization block diagram.

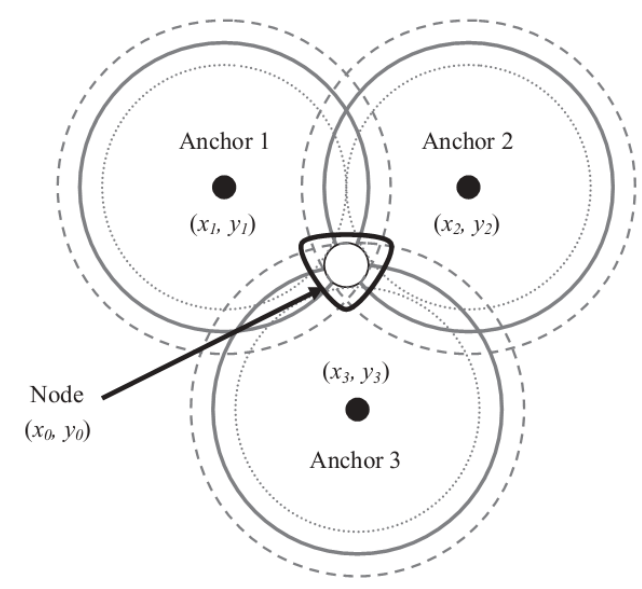

Fig 4: Localization with three anchors.

\section{CONCLUSION}

This paper shows a preview on wireless sensor networks (WSNs) ,application of them and the main problems for this networks like clustering of nodes ,transferring data on this networks, routing the packets in WSNs, localization and security. The main purpose of this paper is to gather information and collect them to build a starting point for who wants to start studying on wireless sensor networks.

\section{REFERENCES}

[1] D. Culler, D. Estrin, M. Srivastava: Overview of Sensor Networks, IEEE Computer Society, August 2004.

[2] Akyildiz, I., Su, W., Sankarasubramaniam, Y., Cayirci, E.: Wireless sensor networks: A survey. Computer Networks39 (4), 393-422 (2002)

[3] Karl, H., Willig, A.: Protocols and Architectures for Wireless Sensor Networks. Wiley, New York (2005).

[4] Sohraby, K., Minoli, D., Znati, T.: Wireless Sensor Networks: Technology, Protocols, and Applications. Wiley, New York (2007).

[5] P. Di Marco: Protocol Design and Implementation for Wireless Sensor Networks, Masters' Degree Project, April 2008,pp 4-21.

[6] B. Wang : Coverage Control in Sensor Networks. Springer, London,2010,pp 4-16.

[7] I.F. Akyildiz , W. Su , Y. Sankarasubramaniam , E. Cayirci: Wire-less sensor networks: a survey, Computer Networks: The International Journal of Computer and Telecommunications Networking, v.38 n.4, p.393-422, 15 March 2002.

[8] A. Sangiovanni-Vincentelli, M. Sgroi, A.Wolisz and J. M. Rabaey:A service-based universal application interface for ad-hoc wireless sensor networks, Whitepaper, U.C.Berkeley, 2004

[9] W. Ye and J. Heidemann: Medium access control in wireless sensor networks, Norwell, MA, USA: Kluwer Academic Publishers, pp. 73 - 91,2004.

[10] IEEE 802.11-Wireless LAN media access control (MAC) and physical layer (PHY) specifications. 1999 
[11] J. Heidemann W. Ye and D. Estrin: Medium access control with co-ordinated adaptive sleeping for wireless sensor networks, IEEE/ACM Transactions on Networking, 12, n. 3 pp. 493-506, 2004.

[12] T.V. Dam and K. Langendoen: An adaptive energyefficient MAC protocol for Wireless Sensor Networks, in SenSys '03. New York, NY, USA: ACM Press, pp. 171180, 2003.

[13] W. R. Heinzelman, J. Kulik, and H. Balakrishnan. Adapti ve protocols for information dissemination in wireless sensor networks. In Proceedings of MobiCom'99, pp. 174-185, Seattle, WA, USA, August 1999.

[14] Haas, Z.J., Halpern, J.Y., Li, L.: Gossip-based ad hoc routing. IEEE/ACM Trans. Netw.14 (3), 479-491 (2006)

[15] Braginsky, D., Estrin, D.: Rumor routing algorithm for sensor networks. In: Proceedings of First ACM International Workshop on Wireless Sensor Networks and Applications, pp. 22-31 (2002)

[16] N. Patwari , J. N. Ash , S. Kyperountas , A. O. Hero , R. L. Moses, and N. S. Correal , "Locating the nodes: Cooperative localization in wireless sensor networks", IEEE Signal Processing Magazine, vol. 22, no. 4, July 2005 , pp. $54-69$.

[17] K. Pahlavan, F. O. Akgul , M. Heidari, A. Hatami , J. M. Elwell , and R. D. Tingley , “ Indoor geolocation in the absence of the direct path ", IEEE Wireless Communications Magazine , vol. 13 , no. 6 , Dec. 2006 , pp. $50-58$.
[18] K. Pahlavan , X. Li, and J. - P. Makela , “ Indoor geolocation science and technology ", IEEE Communications Magazine , vol. 40 , no. 2, Feb. 2002 , pp. $112-118$.

[19] J. Zheng,A. Jamalipour," wireless sensor networks A networking perspective", Wiley ,2009, pp 173-209

\section{AUTHOR'S INFORMATION}

Vahid Houshyarifar was born in urmia,Iran, in 1980. He received the B.S. degree from the Department of Electronics Engineering, K.N.Toosi University of Technology, Tehran, Iran, in 2005. He received the M.S. degree from the Department of Electronics Engineering, Semnan University, Semnan, Iran, in 2007. He is currently a student of PHD degree in communication engineering at University Pardis of Urmia University, Urmia,Iran. His research interests include Wireless sensor networks and its theory.

Mehdi Chehel Amirani received the B.S. degree in Electronic Engineering from Urmia University, Iran, in 1993 and the M.S. and Ph.D. degrees in Communication Engineering from Iran University of Science and Technology (IUST) in 1998 and 2009, respectively. In 2009, he joined the Department of Electrical Engineering at Urmia University, where he currently is an assistant Professor. His research interests include pattern recognition, digital image processing, and secure communication. 